\title{
Quantum hologram of macroscopically entangled light via the mechanism of diffuse light storage
}

\author{
L.V. Gerasimov ${ }^{1}$, I.M. Sokolov ${ }^{1}$, D.V. Kupriyanov ${ }^{1}$, \\ M.D. Havey ${ }^{2}$ \\ ${ }^{1}$ Department of Theoretical Physics, St-Petersburg State Polytechnic University, \\ 195251, St.-Petersburg, Russia \\ ${ }^{2}$ Department of Physics, Old Dominion University, Norfolk, VA 23529 \\ E-mail: kupr@dk11578.spb.edu
}

\begin{abstract}
In the present paper we consider a quantum memory scheme for light diffusely propagating through a spatially disordered atomic gas. The diffuse trapping of the signal light pulse can be naturally integrated with the mechanism of stimulated Raman conversion into a long-lived spin coherence. Then the quantum state of the light can be mapped onto the disordered atomic spin subsystem and can be stored in it for a relatively long time. The proposed memory scheme can be applicable for storage of the macroscopic analog of the $\Psi^{(-)}$Bell state and the prepared entangled atomic state performs its quantum hologram, which suggests the possibility of further quantum information processing.
\end{abstract}

PACS numbers: 34.50.Rk, 34.80.Qb, 42.50.Ct, 03.67.Mn

Keywords: Cold atoms, Light storage and quantum memory, Entangled states 


\section{Introduction}

At present cold atomic systems have shown themselves as promising candidates for effective light storage, see recent reviews of the problem in Refs. [1, 2]. However further improvement of atomic memory efficiencies in either cold or warm atomic vapors is a rather challenging, and not so straightforward, experimental task. In the case of warm atomic vapors any increase of the sample optical depth meets a serious barrier for the electromagnetically induced transparency (EIT) effect because of the rather complicated, and mostly negative, influence of atomic motion and Doppler broadening, which manifest themselves in destructive interference among the different hyperfine transitions of alkali-metal atoms [3]. In the case of cold and dilute atomic gases, prepared for instance in a magneto-optical trap (MOT), for some unique experimental designs an optical depth around several hundred is attainable [4, but there is are certain challenges in accumulation of such a large number of atoms in a MOT and in making such a system controllable.

One possible solution implies special arrangements of effective light storage in a cold atomic sample in the diffusive regime, see [5]. In this case, in an optically dense atomic sample with a given number of atoms, the actual random optical path of light transport becomes much longer than for the single passage of the same sample in the forward direction either under conditions of the EIT effect or in the near resonance transparency spectral window. As a rough estimate, if for an atomic medium formed in a MOT the optical depth on a closed resonance transition is $b_{0}$, then the actual diffusive

path can be $b_{\Sigma} \sim b_{0}^{2}$ i. e. $b_{0}$ times longer. For typical parameters $b_{0} \sim 20-50$ we have a very promising enhancement resource for the light storage via stimulated Raman conversion of the signal pulse as it interacts with the atomic sample in the diffusive regime.

In the present report we discuss such a diffusive quantum memory mechanism in the context of its application to storage of macroscopically entangled light. One experimental technique for generation of polarization entangled light by near subthreshold SPDC type-II light source is well established now, see Ref. [6]. An alternative approach under development utilizes the polarization self rotation effect to generate significantly polarization squeezed light Refs.[7]-[10]. This approach has the substantial advantage of generating quantum states of light with narrow bandwidth and tunability in the vicinity of atomic resonances, important characteristics for an atomic physics based quantum memory. In either case, the generated light possesses the quantum information encoded by some mechanism and, in the case of Ref. [6], in the strongly correlated photon numbers related to different polarization modes. Each mode can be stored in a particular memory unit via transforming the unknown number of photons to the equally unknown number of atoms repopulating the signal level. Importantly, the specifics of the considered memory scheme and of the stored quantum state is that there is no need for further recovering of the signal light in its original mode or modes. We show that all the stored quantum correlations can be observed in the 


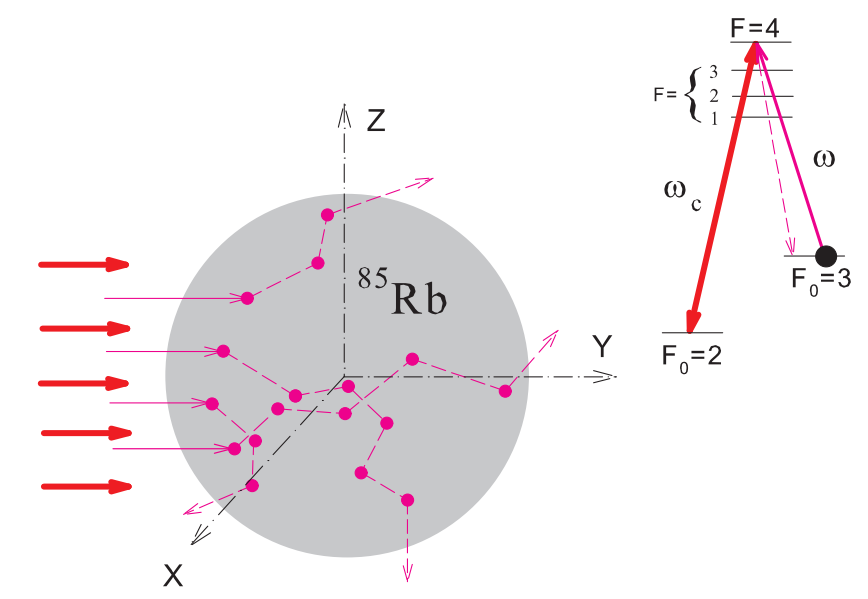

Figure 1. (Color online) The mechanism of diffuse storage of light for the example of light trapping on the $F_{0}=3 \rightarrow F=4$ closed transition in ${ }^{85} \mathrm{Rb}$. The diffuse propagation of a signal mode of frequency $\omega$ is indicated by pink thin lines and arrows. The diffusion process is affected by a strong control mode of frequency $\omega_{c}$ indicated by red and thick arrows. This converts a signal pulse into a long-lived spin coherence in the atomic subsystem.

standard interferometric technique via relevant operations with the prepared quantum hologram.

\section{The mechanism of diffuse light storage}

The considered light storage mechanism is based on stimulated Raman conversion of a signal pulse into a long-lived spin coherence. The main difference with traditional approaches, see Refs.[1, 2], requires that the pulse diffusely propagates through an atomic sample. The hyperfine energy structure of heavy alkali-metal atoms, such as rubidium or cesium, allows convenient integration of these processes Ref.[5]. In Figure 11 we illustrate this through the example of the $F_{0}=3 \rightarrow F=4$ closed transition in ${ }^{85} \mathrm{Rb}$. The crucial point for the protocol is the presence and strong action of the control laser mode repopulating the atoms from the background $F_{0}=3$ hyperfine sublevel to the signal $F_{0}=2$ sublevel via Raman interaction with the upper $F=3,2$ states. As a result, the quantum state of light can be mapped onto a disordered hyperfine coherence and collectivized by the atomic ensemble. Under ideal conditions, without relaxation and atomic losses, the stored state effectively performs a quantum hologram of the signal light, which can survive a relatively long time in the atomic spin subsystem.

To make such a memory scheme feasible, the following two important criteria should be fulfilled. In the Raman mechanism only those pulses can be effectively delayed, which have relatively narrow spectral width

$$
\Delta \omega_{\text {pulse }} \ll \Gamma_{\mathrm{AT}} b_{\Sigma} \sim O(1) \frac{\overline{\Omega_{c}^{2}}}{\Delta^{2}} \gamma b_{\Sigma}
$$


Here $\Gamma_{\mathrm{AT}} \sim O(1) \frac{\overline{\Omega_{c}^{2}}}{\Delta^{2}} \gamma$, where $\gamma$ is the natural radiative decay rate, is an estimate for the bandwidth of the Autler-Townes (AT) resonance, created by the control field. The bandwidth is expressed by the averaged Rabi frequency for the control field $\Omega_{c}$ and by its averaged detuning $\Delta$ from those upper state hyperfine sublevels, which are involved in the Raman process. In our case $\Delta$ can be estimated by the hyperfine splitting $\Delta_{\text {hpf }}$ between the $F=4$ and $F=3$ sublevels in the upper state. The dimensionless optical depth $b_{\Sigma}$ can be estimated as $b_{\Sigma} \sim n_{0}(\lambda / 2 \pi)^{2} L_{\Sigma}$, where $n_{0}$ is a typical density of atoms in the sample, $\lambda$ is the radiation wavelength and $L_{\Sigma}$ is the length of a diffusive path of the signal pulse in the sample, see Figure 1. Physically the condition (11) constrains the spectral domain where dispersion effects are manifestable. However for the spectrally narrow pulses there can be a strong influence of the spontaneous Raman losses initiated by the scattering on the absorption part of the AT resonance. To guarantee that the spontaneous scattering is a negligible effect, the following inequality, as an alternative to (1), should be fulfilled

$$
\Delta \omega_{\text {pulse }} \gg \Gamma_{\text {AT }} \sqrt{b_{\Sigma}} \sim O(1) \frac{\overline{\Omega_{c}^{2}}}{\Delta^{2}} \gamma \sqrt{b_{\Sigma}}
$$

Considered together both the inequalities (11) and (2) leads to an analog of the well known requirement for atomic memory units $b_{\Sigma} \gg 1$, see Ref.[1], i. e. in our case the signal pulse should pass a long transport path in the medium. As we pointed out above, in the diffusive regime this path can be made very long.

The optimal pulse spectrum seems $\Delta \omega_{\text {pulse }} \lesssim \gamma$ which justifies the substantial trapping of light via the multiple scattering mechanism. In this case the variation of the Rabi frequency of the control mode is bounded by the above inequalities, which together comprise the losses and dispersion effects. In reality even a standard one dimensional realization of the quantum memory protocol requires serious optimization efforts, see Ref.[1]. Apparently in the discussed three dimensional configuration, which is principally based on the $D_{2}$-line multilevel energy structure of alkali-metal atom, the optimization scheme is expected to be much more complicated. Inequalities (11) and (2) give us a physically clear but only rough approximation, which we can consider as only the simplest qualitative recommendation. In Figure 2 a typical spectral dependence for the dielectric susceptibility of the atomic sample, modified by the presence of the control mode, is shown. The spectra are reproduced in the vicinity of the $F_{0}=3 \rightarrow F=4$ resonance line of ${ }^{85} \mathrm{Rb}$ and $\Delta$ is the relevant frequency detuning. The sample susceptibility is scaled by the dimensionless density of atoms $n_{0}(\lambda / 2 \pi)^{3}$ and we address the reader to Ref.[5] to see the calculation details. The upper curve indicates the overall "absorption" profile, which is actually responsible not for absorption but for incoherent light scattering, and the lower curves select the contribution of the absorption and dispersion parts of the AT-resonance only. The initial polarization direction for the signal mode $\mathbf{e}$ and the polarization of the control mode $\mathbf{e}_{c}$ are related to the reference frame and the excitation geometry shown in Figure 1. The spectrum of the signal pulse, shown by the pink filled area, is narrower than the original Lorentz profile of the non-disturbed atomic resonance $F_{0}=3 \rightarrow F=4$ but essentially broader than the 


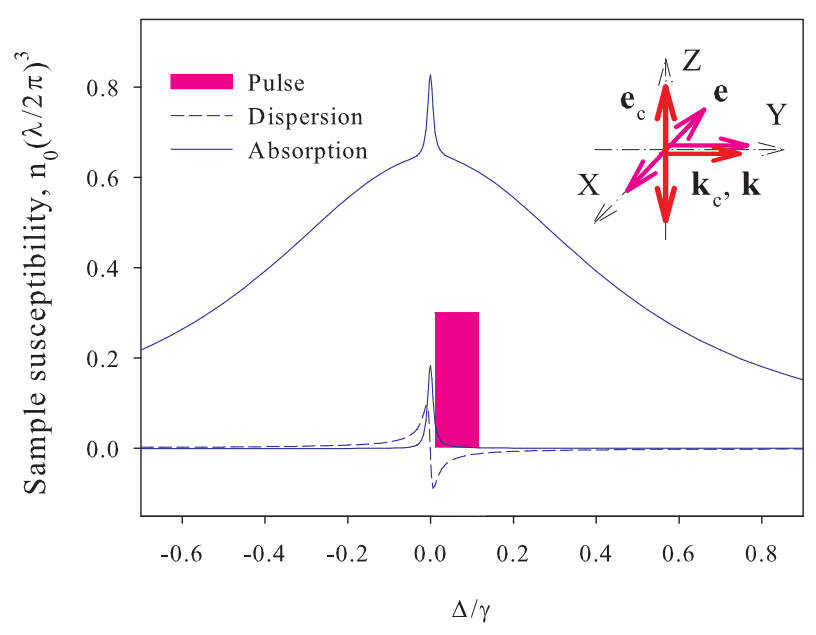

Figure 2. (Color online) Dielectric susceptibility of the sample versus the pulse spectrum. The spectra are shown in the vicinity of the $F_{0}=3 \rightarrow F=4$ resonance line of ${ }^{85} \mathrm{Rb}$ and $\Delta$ is the relevant frequency detuning. The susceptibility is scaled by the dimensionless density of atoms $n_{0}(\lambda / 2 \pi)^{3}$. The upper curve indicates the overall absorption profile modified by the presence of the control mode and the lower curves select the contribution of the absorption and dispersion parts of the Autler-Townes resonance only. The initial polarization and propagation directions for the signal mode $\mathbf{e}, \mathbf{k}$ as well as the polarization and propagation directions of the control mode $\mathbf{e}_{c}, \mathbf{k}_{c}$ are related to the reference frame and the excitation geometry shown in Figure 1 . The pulse spectrum is shown by the pink filled area.

AT resonance. We consider the flat spectral profile to follow how the photons having a frequency uncertainty randomly distributed in the selected spectral area could be potentially delayed via the diffuse memory protocol.

In Figure 3 we demonstrate a portion of our Monte-Carlo simulations of the process. The performed calculations have been done for a spherical atomic cloud consisting of ${ }^{85} \mathrm{Rb}$ atoms with a Gaussian-type radial distribution characterized by a squared variance $r_{0}^{2}$. The optical depth for a light ray propagating through the central point of the cloud is given by $b_{0}=\sqrt{2 \pi} n_{0} \sigma_{0} r_{0}$, where $n_{0}$ is the peak density of atoms and $\sigma_{0}$ is the resonance cross-section for the $F_{0}=3 \rightarrow F=4$ transition. In our calculations we used $b_{0}=20$, which is an example of attainable depth in cold atom experiments with alkalimetal atom samples prepared in a MOT. The graphs of Figure 3 subsequently show how the delay effect associated with the control field is accumulated as the scattering order is increased. In our numerical simulations we assumed the simplest atomic distribution with equal population of all the Zeeman sublevels in the background state $\left(F_{0}=3\right)$. This creates the AT resonance with rather small amplitude, see Figure2. The resonance could be essentially enhanced for the atomic ensemble consisted of the spin oriented atoms, which would select the $\Lambda$-type optical transitions with the highest coupling strength. However even in the case of weak AT resonance for high scattering orders the delay effect becomes quite visible such that a significant part of the light can be stored in the 




Figure 3. (Color online) These graphs subsequently show the delay induced by the control field to those pulse fragments, which are freely passed forward (upper panel), and scattered in the forward direction in the 50-th (central panel) and the 100-th (lower panel) orders of multiple scattering. The gray dashed curve indicates the original profile (with arbitrary scaled amplitude) of the pulse incident on the sample

spin subsystem. As was pointed out in the introduction, for diffusive propagation the light can experience several hundred scattering events before it leaves the sample.

The Monte Carlo simulations normally give a rather realistic approximation of light diffusion to experimental situation but it cannot demonstrate the real potential for the scheme of diffuse light storage in its optimal regime. As known from many discussions of more simple and traditional one dimensional realizations of either EIT or Raman memory protocols, see review [1] and reference therein, the "write-in" and "readout" stages of the protocol are not completely symmetric parts of the entire process. 
The retrieval of the signal pulse can be made much more effective by applying the control mode in the backward direction, a point that is physically supported by time reversal arguments. In particular, for the case of a stimulated Raman process in its optimal configuration the stored signal light can be effectively mapped onto the spin coherence localized near the edge of the atomic sample where light enters the sample. This yields a very effective retrieval in the backward direction, as was confirmed by the round of calculations presented in Ref.[11] for cesium atoms. In the discussed case we can also expect the optimal light conversion to the spin coherence near that boundary of the atomic cloud where the incident light penetrates. Then for the best strategy in the write-in stage of the memory protocol, the optimal spectrum for the signal light should be concentrated near the steep part of the dispersion such that its further retrieval in the forward direction would be ineffective and accompanied by strong spontaneous losses. The time reversal retrieval scheme would be not so easy to organize in the three dimensional disordered configuration, see Ref.[5], and one can use an extremely weak control mode in the read out stage of the protocol to solve the problem and minimize spontaneous losses. In the present report we shall discuss one specific application example where it is not necessary to retrieve the pulse in its original mode and it is only important to provide its effective storage, such that the protocol does not require the light transport throughout the whole sample. For this special situation the above memory scheme is expected to be much more effective than its one dimensional counterpart.

\section{Quantum hologram}

The proposed memory scheme is applicable and adjusted to the situation when the quantum information is originally encoded in the total number of photons in the signal light beam(s), this number considered as a quantum variable. Such variables are insensitive to either spatial or temporal mode structure of the signal light pulse. Physically this means that an unknown number of informative photons can be mapped onto the atomic subsystem via Raman-induced repopulation of the equivalent unknown number of the atoms to the signal level while light diffusely propagates through the sample. Such a situation takes place with storage of the macroscopic analog of the Bell state, this consisting of pairs of photons with either (orthogonal) horizontal (H) or vertical $(\mathrm{V})$ polarizations having unknown but strongly correlated photon numbers, see Ref. [6]. In the present report we shall consider, as an example, the following entangled quantum state of light

$$
\left|\Psi^{(-)}\right\rangle=\sum_{m, n} \Lambda_{m n}^{(-)}|m\rangle_{H 1}|n\rangle_{V 1}|m\rangle_{V 2}|n\rangle_{H 2}
$$

where

$$
\Lambda_{m n}^{(-)}=(-)^{n} \frac{\bar{n}^{\frac{m+n}{2}}}{[1+\bar{n}]^{\frac{m+n}{2}+1}}
$$






Figure 4. (Color online) The quantum hologram of the macroscopically entangled state of light $\left|\Psi^{(-)}\right\rangle$, which can be prepared by a SPDC process, see Ref. [6]. The unknown photon numbers in each polarization are subsequently stored in four memory units.

which possesses completely anti-correlated polarizations in the light beams 1 and 2 , these beams propagating in different directions. The unique property of this state is that detection of a certain number of photons in any polarization (not only in $H$ or $V$ but also in any elliptical polarization state) in beam 1 guarantees the detection of the same number of photons in beam 2 but always in an orthogonal polarization state. In Ref.[6] such a state was called a macroscopic analog of a singlet-type two-particle Bell state. Its Schmidt decomposition, given by Eqs. (3) and (4), can be found via basic expansion for the two mode squeezed state, see Ref. [12]. The quantum state (3) can be parameterized by the average number of photons in each light beam $\bar{n}$.

In Figure 4 we illustrate how the quantum hologram of this light can be realized with the memory units, as described above. Each memory unit stores the photons of a particular polarization and frequency, which diffusely propagate through an atomic cloud and are substantially trapped on the closed transition and the stimulated Raman process is initiated by interaction with the control mode $\omega_{c}$ on other hyperfine sublevels $(F=3,2)$ as is shown in the transition diagram of Figure 1. The more subtle point of this process is that it creates a pure quantum state in the atomic subsystem, which is entangled among the four clouds and has an unknown but strongly correlated number of atoms repopulated onto the signal level $F_{0}=2$ in each cloud, such that their further measurement would demonstrate the presence of quantum non-locality in the matter subsystem.

A standard strategy for a quantum memory normally aims towards a goal of recovery of the signal pulse in its original mode. In our situation there is no need to do that since all the quantum information is encoded into the numbers of repopulated atoms. The observation or detection of these numbers can be organized with the MachZehnder interferometer, as is shown in Figure 4. The interferometer can be adjusted for balanced detection of the signal expressed by the difference of the photocurrents from 


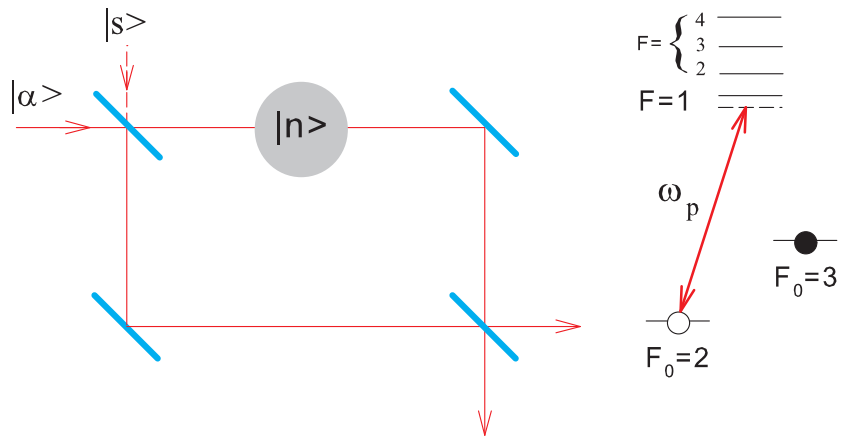

Figure 5. (Color online) Schematic diagram of the Mach-Zehnder interferometer for detecting a small number of atoms stored in a particular cloud. The weak probe coherent mode $|\alpha\rangle$ of frequency $\omega_{p}$ is applied near the resonance of the closed $F_{0}=2 \rightarrow F=1$ transition to avoid effects of Raman scattering. The sensitivity of the interferometer can be enhanced via sending a portion of the squeezed light $|s\rangle$ to the second input port of the interferometer, see Ref. [13].

the output ports. Then the measured signal associated with the small informative phase shift $\delta \phi$, induced in one arm of interferometer, is given by

$$
i_{-}=i_{1}-i_{2} \propto \bar{i} \delta \phi, \quad \delta \phi=\xi n
$$

where the phase shift is proportional to the number of detected atoms $n$ and to a small factor $\xi \ll 1$, which depends on geometry (sample size, aperture of the light beam etc.) and reflects the weakness of the signal. The $D_{2}$-line energy structure of an alkalimetal atom allows one to tune the probe near the resonance associated with the closed transition and to avoid any negative presence of the Raman scattering channel. In the case of ${ }^{85} \mathrm{Rb}$, that is the $F_{0}=2 \rightarrow F=1$ transition. Then the standard sensitivity of the measurement is limited by the shot-noise, Poissonian level, but it can be essentially improved via sending a portion of squeezed light to the second port of interferometer, see Ref.[13].

Let us make the following remark concerning the above detection scheme, which in an ideal situation would perform a certain type of quantum non demolition measurement (QND), see Ref.[14]. At first sight the scheme seems it is specifically related to the polarization basis $|H\rangle$ and $|V\rangle$, which was used in expansion (3) and in the storage protocol shown in Figure 4. However, an identical expansion could be rewritten in any other basis of arbitrary orthogonal elliptical polarizations and this would be described by the same expansion coefficients. In other words the state $\left|\Psi^{(-)}\right\rangle$is insensitive to the type of polarization beamsplitters used for the hologram creation. Then the above QND operation with the hologram can be interpreted as postponed detection of the photons transmitted by the particular beamsplitters. With variation of the beamsplitter types the measurement statistics could demonstrate violation of the classical probability principles. In a particular case of a rare flux consisting of the photons' pairs prepared in a "singlet state" the measurements would show violation of the Bell inequalities. We can also point out that the hologram yields various of interferometric operations and 
could potentially be interesting as a logic element for the further quantum information processing based on a continuous variables scheme.

\section{Acknowledgements}

We thank Maria Chekhova and Timur Iskhakov for fruitful discussions, which initiated this work. The work was supported by RFBR (grant 10-02-00103) and NSF (grant NSFPHY-1068159) and by Federal Program "Scientific and scientific-pedagogical personnel of innovative Russia on 2009-2013" (contract \#14.740.11.0891).

\section{References}

[1] Polzik E, Sørensen A, and Hammerer K 2010 Rev. Mod. Phys. 821041

[2] Simon C et al 2010 Eur. Phys. J. D 58 1-22

[3] Scherman M, Mishina O S, Lombardi P, Laurat J, Giacobino E arXiv:1106.0988v2; Mishina O S, Scherman M, Lombardi P, Ortalo J, Felinto D, Sheremet A S, Bramati A, Kupriyanov D V, Laurat J, and Giacobino E 2011 Phys. Rev. A 83033838

[4] Froufe-Pérez L S, Guerin W, Carminati R, Kaiser R 2009 Phys. Rev. Lett. 102 173903; Guerin W, Mercadier N, Michaud F, Brivio D, Froufe-Pérez L S, Carminati R, Eremeev V, Goetschy A, Skipetrov S I, Kaiser R 2010 J. of Opt. 12024002

[5] Gerasimov L V, Sokolov I M, Olave R G, Havey M D 2011 J. Opt. Soc. Am. B 281459

[6] Iskhakov T S, Chekhova M V, Rytikov G O, Leuchs G, 2011 Phys. Rev. Lett. 106113602

[7] Matsko A B, Novikova I, Welsh G R, Budker D, Kimball D F, Rochester S M, 2002 Phys. Rev. A 66043815

[8] Mikhailov E E, Novikova I, 2008 Optics Lett. 331213

[9] Horrom T, Balik S, Lezama A, Havey M D, Mikhailov E E, 2011 Phys. Rev. A 83053850

[10] Barreiro S, Valente V, Failache H, Lezama A, 2011 Phys. Rev. A 84033851

[11] Sheremet A S, Gerasimov L V, Sokolov I M, and Kupriyanov D V, Mishina O S, Giacobino E, and Laurat J 2010 Phys. Rev. A $\mathbf{8 2} 033838$

[12] Braunstein S L, van Loock P 2005 Rev. Mod. Phys. 77513

[13] Xiao M, Wu L A, Kimble H J, 1987 Phys. Rev. Lett. 59278

[14] Mandel E, Wolf L 1995 Optical coherence and quantum optics (Cambridge University Press) 